\title{
Measurement and Data-Assisted Simulation of Bit Error Rate in RQL Circuits
}

\author{
Quentin Herr, Alex Braun, Andrew Brownfield, Ed Rudman, Dan Dosch, Trent Josephsen, and Anna Herr \\ Northrop Grumman Corp., Baltimore, MD 21240
}

(Dated: 14 April 2021)

\begin{abstract}
A circuit-simulation-based method is used to determine the thermally-induced bit error rate of superconducting logic circuits. Simulations are used to evaluate the multidimensional Gaussian integral across noise current sources attached to the active devices. The method is data-assisted and has predictive power. Measurement determines the value of a single parameter, effective noise bandwidth, for each error mechanism. The errors in the distributed networks of comparator-free RQL logic nucleate across multiple Josephson junctions, so the effective critical current is about three times that of the individual devices. The effective noise bandwidth is only $6-23 \%$ of the junction plasma frequency at a modest clock rate of $3.4 \mathrm{GHz}$, which is $1 \%$ of the plasma frequency. This analysis shows the ways measured bit error rate comes out so much lower than simplistic estimates based on isolated devices.
\end{abstract}

Digital superconducting is a long-standing candidate for beyond-CMOS technology due to high clock rates and unparalleled power efficiency [1]-[3]. Demonstrations of digital functions continue to mature [4], and are currently at the level of small CPUs [5]. Fundamental power advantages derive from both the Josephson junction active devices and non-dissipative interconnects. In contrast to CMOS, superconducting technology is thermally limited not device limited, meaning the devices are sized based on bit-error rate (BER) limitations, not based on lithographic minimum feature size. Current energy-efficient variants of superconducting digital logic, such as RQL and the QFP, have scaled the energy per switching event to within a factor of 100-1000 above Landauer's limit, $\ln (2) k_{B} T$.

The penalty for such performance is more stringent optimization criteria. The circuits need to be characterized not only by timing design, as with CMOS, but also in terms of parametric operating margins and BER performance. Parametric margins are determined using noise-free circuit simulations, but the more relevant question is to determine parametric margins defined in terms of an acceptable BER. For large scale applications, the acceptable BER for individual gates is on order $10^{-24}$. These levels are difficult to access in either simulation or measurement.

Elevated BER can be measured as a function of a tuning parameter, and extrapolated to the region of unobservable levels. This approach has been widely reported [6]-12]. The usual caveats with extrapolation apply, as there are multiple sources of BER in the circuits. Regardless of the error mechanism, BER scales simply with energy set by the device size [1]. While measurement is useful and necessary, a simulation-based approach is also needed.

Models of BER exist for only a few simple cases. Analysis of the comparator [13] predicts how the BER scales with clock rate in the low-speed limit that might be applicable to the QFP [12], but not more generally. Spontaneous switching of biased, isolated junctions is understood [14], but has not been generalized to the distributed networks of SFQ circuits.
Simulation methods are also of limited utility. FokkerPlank simulation has been applied to the comparator [15], but this approach is prohibitive for larger systems. The only alternative is a Monte-Carlo method requiring repeated circuit simulations with random sources for the noise [16]. The method is generally applicable to any circuit schematic, but is low resolution and numerically intensive.

We present a new simulation-based method that is a data-assisted multivariate integration. The method repurposes the integral for parametric yield to find BER:

- Parametric Yield is the integral of the Gaussiandistributed parameters over the operating region of the circuit. This gives the probability of working circuits.

- Bit Error Rate is the integral of the Gaussiandistributed noise currents over the operating region of the circuit. This gives the probability of working clock cycles.

A single free parameter, effective noise bandwidth, is determined for each scenario by comparing simulation to measurement. The approach is generally applicable to any circuit schematic and any noise-induced error mechanism associated with Single-Flux-Quantum (SFQ) circuits. The method is deterministic and can calculate arbitrarily small error probabilities with high resolution. The method is efficient in terms of circuit simulations, requiring about $3^{N}$ margin calculations for $N$ noise sources. Noise sources are included no more than eight at a time, but a sliding window of inclusion covers larger circuits.

BER simulation can be used to improve parametric optimization in the design phase. An important feature here is to ability to calculate individual margins (and BER) as an intersection across global parametric corners. At each corner parameter set, margins are calculated in parallel, and the final number is the most pessimistic result. This approach could be used to calculate the most pessimistic BER across global parameter mistargeting, or across signal input timing. This is adjacent to the statistical timing analysis of advanced-node CMOS, see e.g. [17]. 
The rest of the paper covers simulation-based numerical evaluation of the multivariate Gaussian integral, simulated BER of two representative logic gates, and measured BER and comparison to the simulation. The dataassisted method uses a generic value for effective bandwidth in the initial simulations, and then solves for the measured values. The discussion centers on the physical meaning of the effective noise bandwidth.

\section{MULTIVARIATE GAUSSIAN INTEGRAL}

BER is the probability of failure, which is given by the complement of yield. This is the integral over the tails of the Gaussian distribution, outside the operating region of the circuit. We start with the one dimensional case and generalize to higher dimensions. In the simplest case the upper and lower margins of the circuit are equal. Normalized margin $r=m / \sigma$ is the circuit operating margin $m$, normalized in units of the standard deviation. In this case

$$
\operatorname{yieldc}(r)=\operatorname{erfc}(r / \sqrt{2})
$$

The upper and lower margins, $r_{a}$ and $r_{b}$, are likely different. If so, the contributions can be computed separately, normalized, and summed.

$$
\operatorname{yieldc}\left(1, r_{a}, r_{b}\right)=1 / 2 \operatorname{erfc}\left(r_{a} / \sqrt{2}\right)+1 / 2 \operatorname{erfc}\left(r_{b} / \sqrt{2}\right)
$$

In $N$ dimensions, analytic results obtain if the operating region is defined by a radius $r$.

$$
\operatorname{yieldc}(N, r)=\mathrm{Q}\left(N / 2, r^{2} / 2\right)
$$

where $\mathrm{Q}(a, x)=\Gamma(a, x) / \Gamma(a)$, is the normalized upper incomplete gamma function as defined e.g. in [18]. This is also known as the regularized gamma function. Here the limits of integration correspond to a constant value of the Gaussian joint probability distribution, which has radial symmetry.

The operating region of the circuit generally does not have radial symmetry and must be mapped out. This is illustrated for two dimensions in Fig. 1, and generalizes to higher dimensions. We approximate the operating region by connecting boundary points with simplexes. Each simplex $s$ gives a yield estimate based on the mean value $\left\langle\mathrm{Q}_{s}\right\rangle$ of the $Q$ values evaluated at the boundary points. This estimate is weighted by the angle $\Omega_{s}$ subtended by the segment. The integral is approximated by a normalized Riemann sum of these terms. In $N$ dimensions,

$$
\text { yieldc }=\frac{1}{\Omega_{N}} \sum_{s} \Omega_{s}\left\langle\mathrm{Q}_{s}\right\rangle
$$

which is normalized to the total angle $\Omega_{N}$. The mean value of $\mathrm{Q}$ for each simplex is

$$
\left\langle\mathrm{Q}_{s}\right\rangle=\frac{1}{N} \sum_{i=1}^{N} \mathrm{Q}\left(N / 2, r_{t[s, i]}^{2} / 2\right)
$$

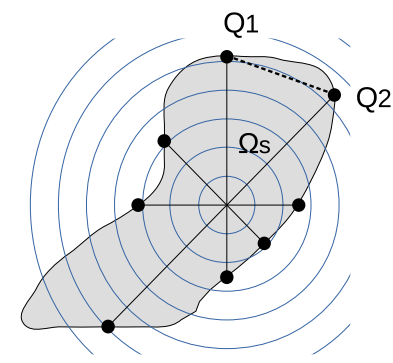

FIG. 1. The operating region of the circuit, shaded, overlays the Gaussian joint probability distribution, shown as concentric isocontours equally spaced in units of sigma. The operating region is approximated by points on the operating boundary, organized as simplexes (line segments in the two dimensions shown). The simplex defined by points Q1 and Q2 subtends an angle $\Omega_{s}$. The mean value of the Q-function for these points, weighted by the angle, is one term in the Riemann sum that approximates the Gaussian integral over the operating region.

Simplexes are indexed by $s$. For a given simplex, $r_{t[s, i]}$ returns the value of radius, indexed by $i$, for each of the points.

Details of the algorithm are described in Appendix 1. Including calculation of the angle $\Omega_{s}$, the annealing schedule of the adaptive algorithm that adds new points to the operating boundary, and simplex fracturing to create a finer grid.

The algorithm does not require the operating region to be convex, which is a fundamental constraint in simplicial design centering [19, 20]. Here it is only required that the operating region be single-valued as viewed from the origin, which corresponds to the binary search vectors. The value of the integral is dominated by regions of the operating boundary nearest the origin. This implies that the computational intensity is similar to finding global minima by exhaustive search. In practice, covering the space exhaustively requires about $3^{N}$ margin calculations. This can be understood as the set of all search vectors for which each dimension has a value of $+1,-1$, or 0 . This set captures all of the potential dependencies of the space and of all subspaces. The number of simplexes grows rapidly as points are added, which currently imposes a practical limit of about eight dimensions.

\section{BER SIMULATION}

In simulation, an auxiliary dc current source is applied across each junction in the circuit. The current through each source is a circuit parameter, with nominal value set to zero and sigma equal to rms noise current,

$$
I_{\mathrm{rms}}=\sqrt{\frac{4 k_{B} T I_{c} \beta}{\Phi_{0}}}
$$

which scales with junction critical current, $I_{c}$. The expression derives from the Johnson noise, $\sqrt{4 k_{B} T B / R}$ us- 
ing the junction characteristic impedance, $R=\sqrt{L_{J} / C_{J}}$, plasma frequency, $B=1 /\left(2 \pi \sqrt{L_{J} C_{J}}\right)$, and inductance, $L_{J}=\Phi_{0} /\left(2 \pi I_{c}\right)$. The bandwidth $\beta$ is a unitless fraction of the plasma frequency. We will use the effective bandwidth $\beta$ as a fitting parameter to relate the simulation result to measured data. This expression for noise current does not depend on junction capacitance, $C_{J}$ or shunt resistance.

The overall method is 1) choose an initial value for $\beta$ to simulate BER, 2) compare the simulated result to measured data to find the experimentally-derived effective $\beta$ for each error regime, and 3) use this value in subsequent simulations. All BER simulations in this paper used the initial value $\beta=0.25$.

On the way to evaluating the Gaussian integral, the algorithm reports the critical search vector, directed towards the point on the operating boundary found to be nearest the origin. This indicates which sources contributed most to nucleating the error. The critical vector is suggestive but not definitive. A gate e.g. with symmetric inputs would have equally critical vectors on both, but only one could be reported. However, the algorithm would integrate over all paths to calcualte BER.

It is intractable and unnecessary to include all noise sources at once in the BER simulation. Instead, it is enough to include sources that contribute to nucleation of the error. Simulations proceed with inclusion 7-8 noisecurrent parameters at a time, chosen among contiguous junctions. We use a sliding window, meaning that the parameter-inclusion sets overlap. Each window returns a BER. The most relevant window is the one that returns the highest BER.

We applied the algorithm to the RQL circuits shown in Fig. 2. For present purposes, the AND gate is representative of gate implementations that do not have a Josephson comparator, and the DFF serves as a diagnostic for comparator-based gates, including RSFQ and the QFP. The AND gate 21] is based on three-input majority, with one input tied to ground. This is analogous to QFP logic, but implementation in terms of data encoding, interconnect, and clock phasing are distinct. The RQL implementation is low-latency as it does not require advance of the clock phase to prevent back-traveling pulses on the inputs 22]; multiple gates can be cascaded on a single phase. The D flip-flip (DFF) stores the input until it is clocked out through the comparator. The implementation is similar to the RSFQ DRO. However, both positive and negative polarity pulses are used in RQL, so the stored signal may be of either polarity. The utility of the DFF and other features of the RQL library are beyond the scope of BER considerations, and will be described elsewhere.

The BER calculation at each set point requires a few thousand binary searches across multiple windows. However, simulations can proceed in parallel, so full characterization of the gate requires less than 24 hours of real time using a few large servers running WRspice [23].

Simulated BER of the AND gate is shown Fig. 3 a.
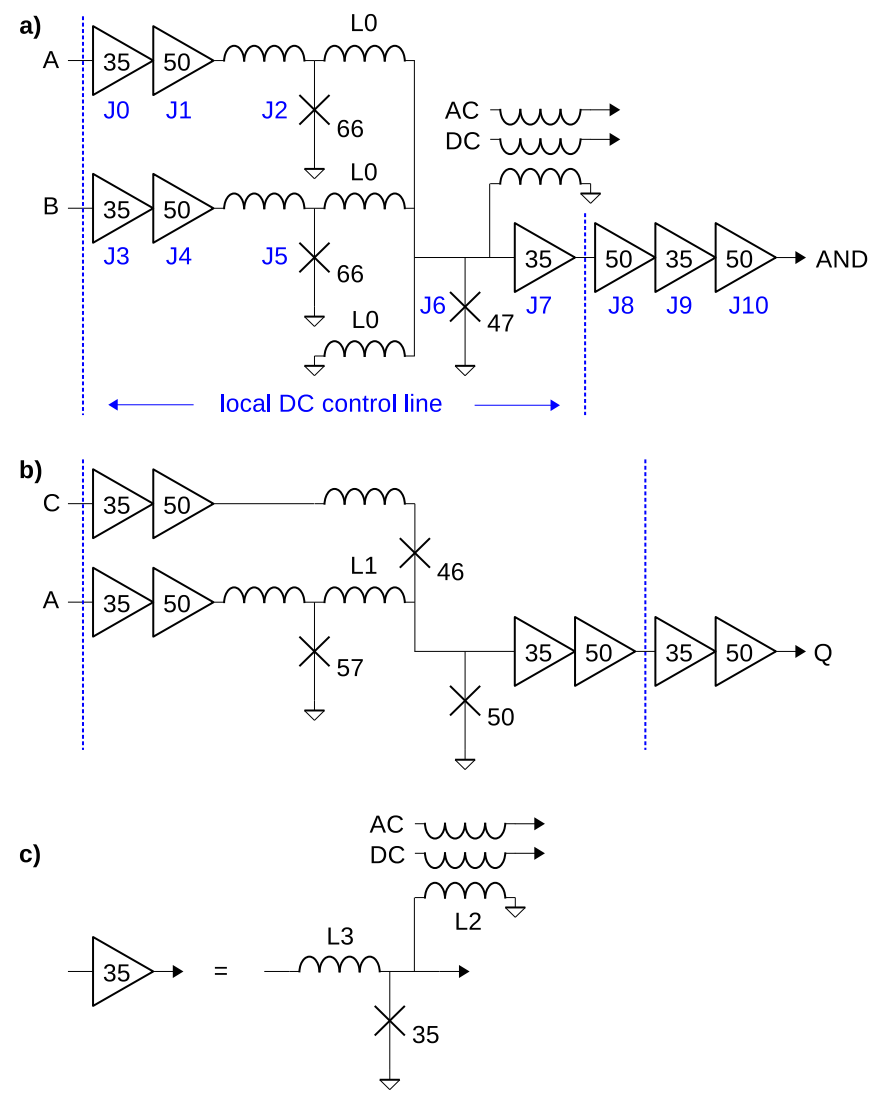

FIG. 2. Circuit schematics are shown for a) the AND gate, b) the DFF gate, and c) the JTL subcircuit. The critical currents of the junctions, $I_{c}$ are given in units of $\mu \mathrm{A}$. JTL junctions are biased with an $\mathrm{AC}$ sinusoid and a DC offset via mutual inductance to L2. The signal inductors, including L3, typically have values ranging from $0.33-0.5 \Phi_{0} / I_{c}$. The storage inductors L0 and L1, and the bias inductors L2, are larger. The AC bias is applied globally, including the input and output amplifier stages. The DC bias is applied locally to the device under test via a separate control line.

Three sets of curves are visible; the progression with negative slope in the left, and two progressions of positive slope on the right. All of this can be expressed analytically as the sum of three error functions, with fitting coefficients that depend on the AC amplitude, A. The fit, detailed below, produced the contour plot shown in Fig. 3r.

The three-sided contours correspond to typical JTLbased error mechanisms. The DC offset optimally produces symmetry between positive and negative SFQ pulses by putting a $\Phi_{0} / 2$ flux bias on the bias inductor (L2 in Fig. 22). AC amplitude powers the transistions. When DC is low (side a), positive pulses fail to propagate and are annhiliated by the trailing negative pulse. When $\mathrm{DC}$ is high and $\mathrm{AC}$ is low (side b), it is the trailing negative pulse that fails to propagate. When DC and AC are both high (side c), positive pulses are spontaneously generated in error. By symmetry, one might expect that DC low and $\mathrm{AC}$ high would produce a fourth regime where 
TABLE I. Critical vectors for the points indicated in the AND gate simulation of Fig. 3a, illustrating three different error mechanisms. Columns correspond to noise sources on the junctions of Fig. 2a

\begin{tabular}{cc|rrr|rrr|rrrrr} 
& & \multicolumn{4}{|c|}{ Input A } & \multicolumn{5}{|c|}{ Input B } & \multicolumn{5}{c}{ Output } \\
Point Window & \multicolumn{1}{|c}{ J0 } & \multicolumn{1}{c|}{ J1 } & \multicolumn{1}{c|}{ J2 } & J3 & J4 & J5 & J6 & J7 & J8 & J9 & J10 \\
\hline $\mathrm{a}$ & 1 & -0.271 & -0.924 & -0.271 & 0.000 & 0.000 & 0.000 & 0.000 & 0.000 & - & - & - \\
$\mathrm{b}$ & 1 & 0.197 & 0.558 & 0.197 & 0.279 & 0.674 & 0.279 & 0.000 & 0.000 & - & - & - \\
$\mathrm{c}$ & 2 & - & - & 0.163 & - & -0.000 & 0.543 & 0.774 & 0.231 & 0.163 & 0.000 \\
\hline
\end{tabular}

negative pulses were generated in error. The asymmetry is in the data encoding. Every positive pulse is followed half a cycle later by a negative pulse that serves as a reset. Spontaneous negative pulses that anticipate the transistion do not register as an errors. This extends the operating region of the circuit up and to the left well beyond normal design limits.

In the AND gate, the three error mechanisms may arise in different parts of the circuit. This is illustrated for the three points (a), (b), and (c) called out in Fig. 31. The critical vectors for these points are entered in Table I] Point (a) is centered on one of the input JTLs, point (b) straddles both inputs, and point (c) is centered on the output. For each case, the table shows the dominant sliding window of parameter inclusion.

It would be possible - but needlessly intensive - to run and rerun the BER simulations for different values of effective bandwidth in order to match the experimentally measured result. Instead, we develop a functional fit to the simulations that incorporates the bandwidth dependance. For each value of $A$, the simulation points were partitioned and fit to one of three error functions, using margin and noise coefficients. The total BER is given as the sum of these three terms.

$$
\begin{aligned}
& \operatorname{BER}\left(x, A, \beta_{a}, \beta_{b}, \beta_{c}\right)=\frac{1}{2} \operatorname{erfc}\left[\frac{x-m_{a}(A)}{\sqrt{2 \beta_{a}} n_{a}(A)}\right] \\
& +\frac{1}{2} \operatorname{erfc}\left[\frac{m_{b}(A)-x}{\sqrt{2 \beta_{b}} n_{b}(A)}\right]+\frac{1}{2} \operatorname{erfc}\left[\frac{m_{c}(A)-x}{\sqrt{2 \beta_{c}} n_{c}(A)}\right]
\end{aligned}
$$

The three similar terms fit the (a), (b) and (c) curves. For the first term, the fitting parameter $m_{a}(A)$, corresponds to noise-free margin which determines the offset along the $x$-axis. The term $\sqrt{\beta_{a}} n_{a}(A)$ corresponds to rms noise, with the dependance on effective noise bandwidth, $\beta_{a}$, made explicit. All of the curves are well-characterized by a single function using fitting coefficients that are linear functions of $A$.

$$
m_{a}(A)=A m_{1 a}+m_{0 a}, \quad n_{a}(A)=A n_{1 a}+n_{0 a}
$$

Fit values for the three curves are entered in Table II] Note that the slopes of curves (a) and (b) are constant across the range of $A$ values. The resulting function

$$
\operatorname{BER}\left(x, A, \beta_{a}=0.25, \beta_{b}=0.25, \beta_{c}=0.25\right)
$$

is a good fit to all of the simulation points, as shown in Fig. 3b. The exception is that the positive-slope progressions for $A=\{1.188,1.259\}$ are above the line. Further
TABLE II. Coefficients for the function of Eqn. 1 fit to the AND gate simulation points.

\begin{tabular}{r|rr|rr} 
& $m_{1_{-}}$ & $m_{0_{-}}$ & $n_{1_{-}}$ & $n_{0_{-}}$ \\
\hline $\mathrm{a}$ & -7.724 & 7.542 & 0 & 0.1760 \\
$\mathrm{~b}$ & 7.732 & -1.650 & 0 & 0.1240 \\
$\mathrm{c}$ & -10.173 & 16.151 & 0.0655 & 0.1480 \\
\hline
\end{tabular}

investigation showed that these were over-bias errors in the output JTL, far from the gate itself. These errors are real, but are outside of the gate characterization.

We applied the same method to BER simulation of the DFF, shown in Fig. 4. Here the waterfall curves are interrupted by a noise floor at about $10^{-40}$. Further analysis showed that the floor corresponds to decision errors in the comparator, while the waterfalls corresponds to errors in the JTLs surrounding the gate. The floor is far below the limits of direct observation in measurement. This simulation indicates that the AC amplitude and DC offset set points, applied directly to the JTLs but not to the comparator itself, do not allow exapolation to the dominant error mechanism at low BER. What is needed is an auxillary current bias applied directly to the central node of the comparator [6, 13], as this would produce a large shift in comparator threshold.

\section{BER MEASUREMENT AND COMPARISON TO SIMULATION}

Integrated circuits containing the RQL gate library were designed into a six-metal-layer fabrication process supplied by D-Wave 24]. This revision of the fab featured Josephson junctions with $100 \mu \mathrm{A} / \mu \mathrm{m}^{2}$ critical current density. The logic gates used individual circuits with dedicated input stages and output amplifiers on-chip. The circuits shared a global $3.4 \mathrm{GHz}$ resonant clock network that provisioned the entire active area of the chip.

Crosstalk between the clock and signal lines in the pressure-contact LHe dip probe was a challenge, so the output data link was established using differential signals, amplified by a pair of Miteq JSMF4-02K180-30-10P LNAs at the probe head. $3 \mathrm{~dB}$ attenuators $(50 \Omega)$ at the inputs of the LNAs were used to improve the impedance match. These fed a Marki BAL-0026 balun that produced a single-ended signal for the lab instruments. A Keysight J-BERT M8020A produced the data patterns and counted the errors. This was synchronized to a pair 

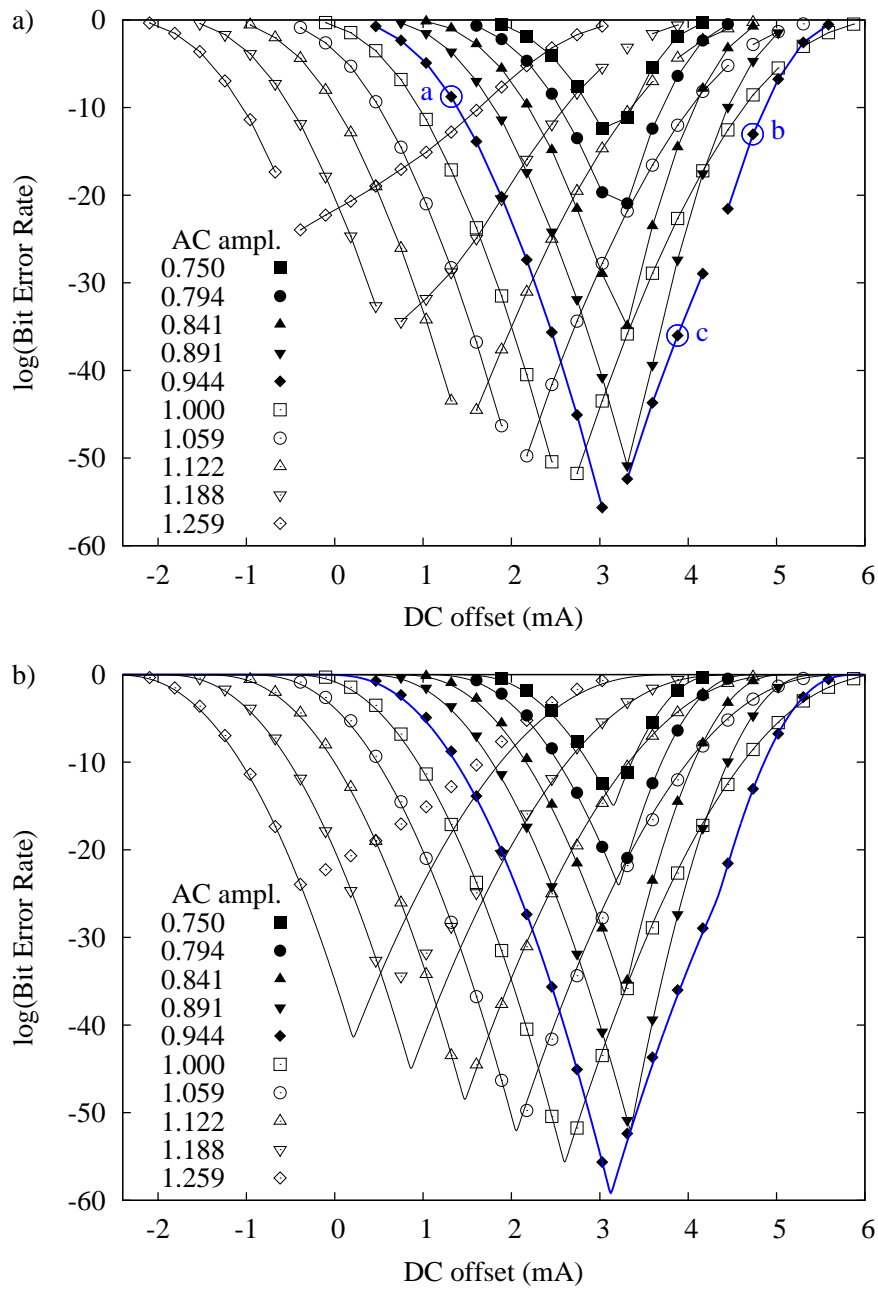

c)

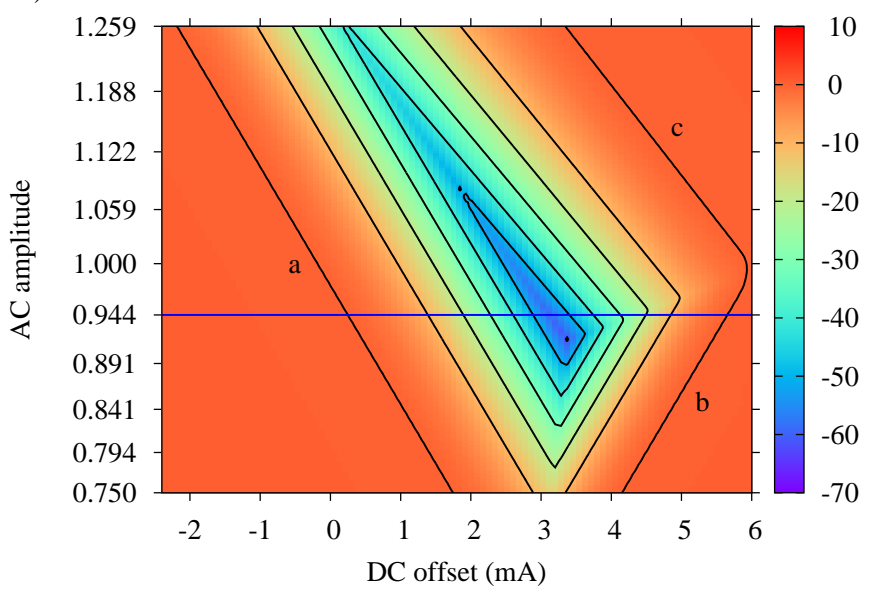

FIG. 3. Simulated BER of the AND gate as a function of DC level, $x$, and normalized AC amplitude, $A$. a) Simulations (points) for each value of $A$ are connected with lines to guide the eye. Gaps in the line are where the maximum BER moved from one sliding window to the other. Two sliding windows were used for inclusion of the noise current sources. The points (a), (b), and (c) are called out for further analysis. b) Simulated points replotted, now with curves defined by Eqn. 2 that captures the result in functional form. c) The function of Eqn. 2 plotted as a contour map. The outer contour is for a BER of 0.5, which equates to noise-free margins. The horizontal blue line marks a cross-section corresponding to the blue line in the upper panels. Each contour has three sides (a), (b), and (c) representing three distinct error mechanisms.

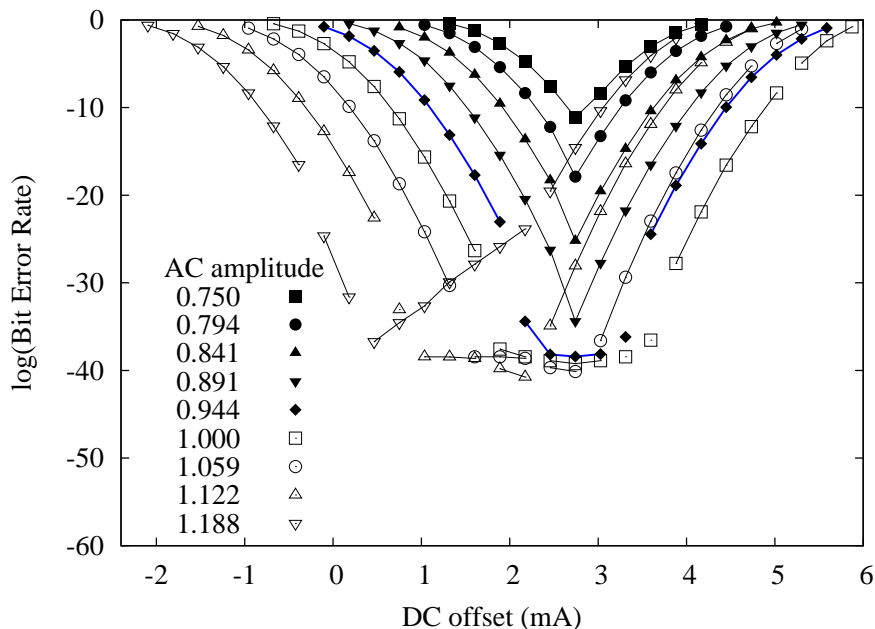

FIG. 4. Simulated BER for the DFF gate as a function of the local DC level and normalized AC amplitude, $A$. Four sliding windows were used, centered on each of the inputs, the output, and the gate itself. Points for each value of $A$ are connected with lines. Gaps in the line are where the maximum BER moved from one window to the other. All four windows are represented in the data for $A=0.944$ (bold lines). The noise floor arises from switching errors in the gate comparator.

of Rohde \& Schwarz SGS100A sources that powered the clock network with I and Q signals. DC levels were generated by a Stahl BS 1-10.

Measurement of five different logic gates is shown in Fig. [5 . The AND and OR gates are closely related as they derive from the same majority gate. For the OR, the third input is initialized to logical "1." The Inverter and XOR [25] are also closely related; the Inverter is derived from XOR, with one of the inputs initialized to logical "1." BER for AND and OR are coincident on the left, but offset on the right. BER for the Inverter and XOR are nearly coincident on the right, but offset on the left. These offsets might be inherent to the design and test vectors, or due to parametric variations in fab or trappedflux effects in test. Additional simulations and test would be needed to resolve these questions. The main result is that the slopes of the curves are consistent, reflecting that the observed errors are induced in interconnect JTLs, which are similar among the designs.

Measured BER of the AND gate at different AC and DC set points, shown in Fig. 5b, mirrored the simulations. Curves fitting the simulation to the measurement, also shown, used the function defined in Eqn. 1 with arguments

$$
\operatorname{BER}\left(x=1.04 x^{\prime}, A=0.99 A^{\prime}, \beta_{a}=0.058, \beta_{b}=0.14, \beta_{c}=0.23\right)
$$

where $x^{\prime}$ and $A^{\prime}$ are the DC offset and AC amplitude scale for the measured data. These numbers produce good quantitative agreement. The factor of 1.04 indicates a small registration error on DC offset. This could be ac- 

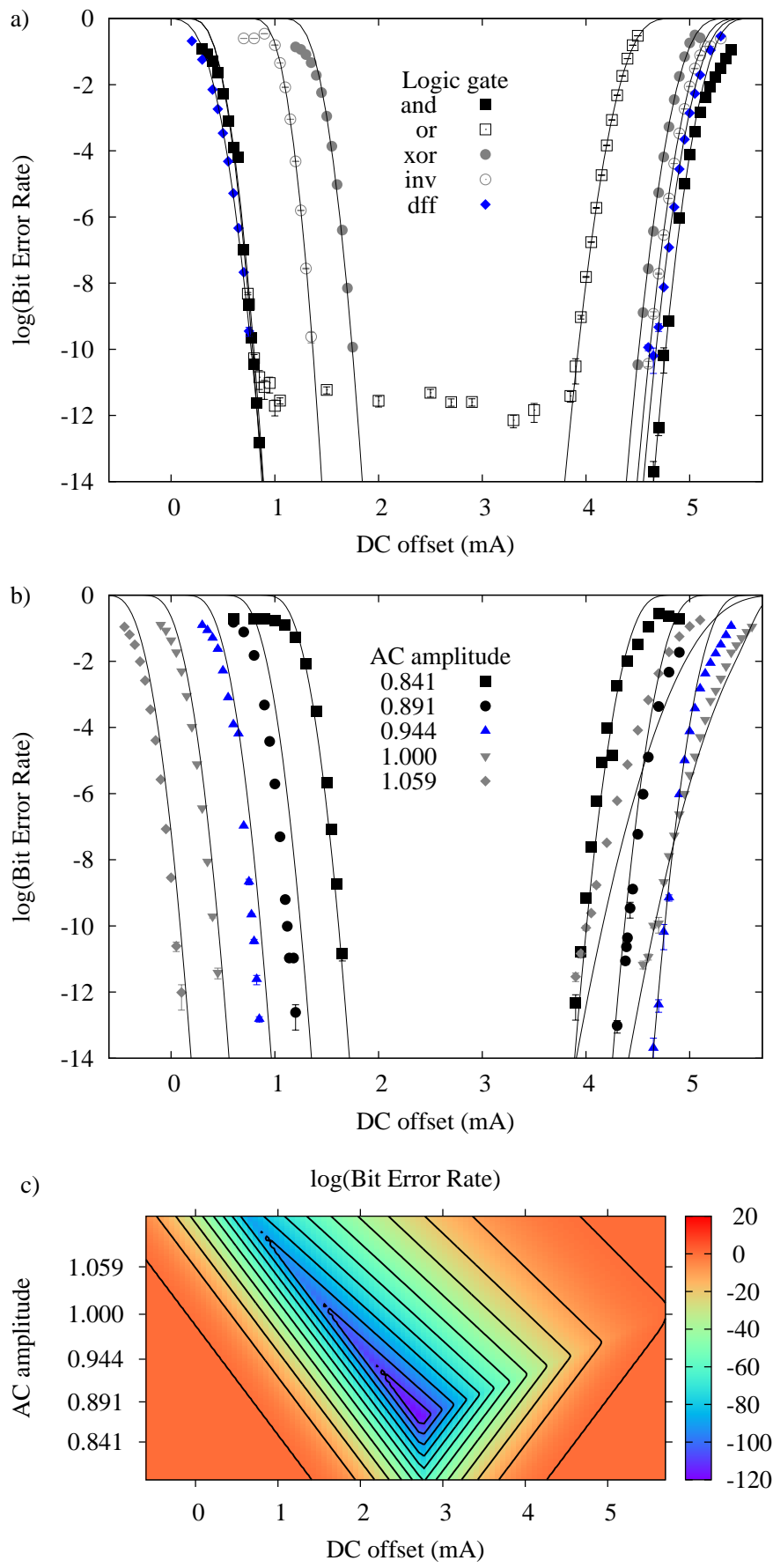

FIG. 5. Measured BER (points) as a function of DC offset. Error bars visible for the lowest points correspond to binomial counting statistics. a) Five different logic gates measured at an AC amplitude, $A=0.944$. Each curve is fit to an error function to guide the eye. A noise floor is visible for the OR gate. We attribute this to observed limitations of the output data link for this particular case, and not to the gate itself. b) Additonal measurements of the AND gate at several values of $A$. Measurements are fit to the simulation result (lines) via Eqn. 3 c) The function of Eqn. 3 plotted as a contour map. The outside contour is for a BER of 0.5 , corresponding to noise-free margins. The inside contour is for $0.5 \times 10^{-110}$. counted for by a $4 \%$ shift in mutual inductance to the control line. The AC amplitudes for the measurement have an arbitrary scale, relative to simulation, but the spacing of $0.5 \mathrm{~dB}$ is fixed for both. A single scaling factor, 0.99 , aligned the simulation to the measurement. These are small disparities given that the simulation used nominal design values; no attempt was made to incorporate global parametric targeting. The primary result lies in the values for effective noise bandwidth. These numbers were chosen to be 0.25 in simulation, but came out widely divergent - and generally smaller - in measurement.

Measured BER of the DFF were similar to the AND gate. This is unsurprising, as simulation indicates that observable errors belong to the JTLs, not the gate itself. However, the DFF comparator produced a BER floor in simulation. To induce comparator errors at observable levels, an additional detuning bias would be needed that directly shifts the comparator threshold. We would expect the effective noise bandwidth for comparator errors to be higher than the other error mechanisms, as the error does not depend on junction switching time per se, but on a shorter time scale when the phase difference between the junctions passes some threshold. Based on a review of previous work [13, 15], an effective normalized bandwidth of 0.5 is our best estimate. If so, the simulations using a bandwidth of 0.25 underestimated comparator errors, and the BER floor is actually around $10^{-25}$ based on scaling given in Eqn. 1 .

\section{DISCUSSION}

Results of the previous section show that 1) operating margins at low BER are generally wide enough to support complex RQL circuits. 2) BER in measurement is less than in simulation, if effective noise bandwidth is taken to be the Josephson junction plasma frequency, and 3) the effective noise bandwidth depends on the error mechanism.

AC overbias errors, corresponding to curves (c) in Figs. 3] and 5, involve spontaneous junction triggering that can be modeled using the Kramers escape rate. This analysis gives a first-principles estimate without resort to an effective noise bandwidth. The Kramers escape rate is $f \approx f_{a} \exp \left[-\Delta U /\left(k_{B} T\right)\right]$, where $k_{B} T$ is the thermal energy, $\Delta U$ is the potential barrier, and $f_{a}$ is the attempt frequency. Here we can safely model the noise as purely thermal at $4.2 \mathrm{~K}$, because DC and AC currents are very weakly coupled to the circuit using magnetic transformers. External noise scales as $k^{2}$, where $k$ is coupling coefficient [1].

The potential barrier for a single biased Josephson junction is approximately [26]

$$
\Delta U\left(I_{b}\right) \simeq \frac{\Phi_{0}}{2 \pi} \frac{2 I_{c}}{3}\left(2-\frac{2 I_{b}}{I_{c}}\right)^{3 / 2}
$$

where the total bias current through the junction is the sum of AC and DC components, $I_{b}=I_{\mathrm{DC}}+I_{\mathrm{AC}}$. At the 
nominal value $I_{\mathrm{DC}}=2.8 \mathrm{~mA}$, the externally-applied $\mathrm{DC}$ offset current produces a bias current of only $I_{b}=0.24 I_{c}$ through the junction. This gives the transfer function $I_{b} / I_{c}=0.086 x / \mathrm{mA}$. The $\mathrm{AC}$ current $I_{\mathrm{AC}}$ shifts the BER function by an arbitrary offset-for comparing to measured data this is left as a fitting parameter. The resulting Kramers escape rate is

$\operatorname{BER}(x) \simeq \alpha \exp \left[\frac{\Phi_{0} I_{c}}{3 \pi k_{B} T}\left(2-2\left[0.086 I_{D C}+I_{A C}\right] / \mathrm{mA}\right)^{3 / 2}\right]$

where the prefactor $\alpha=10$ is the number of attempts per clock cycle. This value does not need to be precise for low BER values. The function corresponds to the asymptotic form of erfc, and is valid in the region of low BER. Fitting the measured data for overbias errors (the steeper curves on the right of Fig. 5b) with Eqn. 4. using critical current and AC bias as fitting parameters, results in $I_{c} \approx 110 \mu \mathrm{A}$.

The effective critical current indicated by the Kramers escape rate is about three times larger than the critical current of the individual junctions used in a circuit, suggesting that the error mechanism is distributed across multiple junctions. This result comports nicely with the simulation result shown as curve (c) in Table@with about three junctions involved in the nucleation of overbias errors in the output JTL. The JTL inductances have an $I_{c} L$ product of about $\Phi_{0} / 3$, meaning that the three JTL junctions span about one- $\Phi_{0}$ of interconnect.

The normalized effective bandwidth for overbias errors in measurement, $\beta_{c}=0.23$, agrees well with initial simulations with $\beta_{c}=0.25$ and indicates a spontaneousswitching time that is long compared to the plasma frequency. This simply means that junction switching time is longer at low values of overbias, as observed in [27].

Annihilation errors, corresponding to curves (a) in Figs. 3 and 5, have a different character. These errors arise when the leading, positive pulse in the RQL data encoding fails to propagate and is annihilated by the trailing negative pulse. Since the positive and negative pulses are separated in space and time by a half-cycle, the noise must persist for a fair fraction of a clock cycle in order to produce an error. This implies much lower effective noise bandwidth. The measured value, $\beta_{a}=0.058$ corresponds to about $17 \%$ of the clock cycle, or $67 \%$ of the quarter-cycle of the four phase clock at $3.4 \mathrm{GHz}$ clock and $340 \mathrm{GHz}$ plasma frequency. Since noise bandwidth for annihilation errors is determined by clock rate it is expected to increase with clock rate, to be confirmed in future experiments.

Similar arguments exist for annihilation error corresponding to curve (b) in Fig. 3-5, but here the trailing, negative pulse of the RQL data encoding fails to propagate and is annihilated when there is a positive pulse on the next cycle. Different effective bandwidths between curves (a) and (b) is unsurprising as the data encoding is asymmetric, and as the errors arise in different parts of the circuit, based on the critical vectors entered in Table 1

\section{CONCLUSION}

Here we have presented a framework to explain, predict, and quantify the BER of RQL gates. The integral of the Gaussian-distributed noise currents associated with the Josephson junctions is evaluated across the multidimensional operating region of the circuit. Measurement determines the value of a single parameter, effective noise bandwidth, for each error mechanism, in a data-assisted approach. All this would apply equally well to the other superconducting SFQ logic families.

The BER in combinational RQL gates such as the AND gate is governed by the JTL-based error mechanisms, including spontaneous switching at overbias and pulse annihilation at underbias. The effective noise bandwidth is quite low for pulse annihilation errors, as it is based on the clock rate, not the junction plasma frequency. The distributed nature of the SFQ pulse means that multiple junctions are involved in nucleating the error for spontaneous switching, so the effective device size is that of multiple devices in parallel, in agreement with [14]. All this explains why the measured BER extrapolates to $10^{-110}$, which is much lower than predicted by simplistic estimates for isolated devices. In the longjunction limit [28], JTL interconnect can be virtually dissipation free while maintaining negligible BER, as "the coupling between mechanical and thermal modes vanishes" 29]. Ultimately this may lead reversible logic gates as well [30, 31].

The clocked comparator of the RQL DFF gate is representative of the other SFQ logic families, including RSFQ and the QFP. BER simulation showed that the comparator produced a noise floor, at about $10^{-25}$ using junctions with $50 \mu \mathrm{A}$ critical current, as the comparator does involve isolated junctions and higher noise bandwidths. This BER is still quite low, suitable for petaflop-scale computing, but larger junctions would be needed in order to maintain this level while taking the parametric spread of individual junctions into account. For both the DFF and the AND gate, extrapolated BER of $10^{-25}$ or better is maintained across an AC power range of $3.6 \mathrm{~dB}$, equivalent to $\mathrm{AC}$ amplitude margins of $\pm 20 \%$.

Overall we conclude that the BER in RQL circuits is sufficiently low for large-scale applications, even with junction critical current scaled down to $35 \mu \mathrm{A}$ minimum in the JTLs, as predicted [1]. BER performance of each gate in the library can characterized and optimized by appropriate device sizing in the design phase, using BER simulation as the primary tool.

\section{APPENDIX}

We now describe some details of the numerical integration of the Gaussian distribution across the operating region of the circuit. As discussed above, mapping out the operating region is effectively to find the global minimum by exhaustive search. If only the local minimum 
were wanted, the downhill simplex method could be used. Instead the entire operating region is mapped out with simplexes. The algorithm uses an anneal to first search exhaustively, and then to adaptively apply higher resolution to the regions of interest.

The algorithm is as follows:

1. Calculate all $2 N 1 \mathrm{D}$ margins and all $2^{N}$ corner margins

2. Make the initial simplexes, each using $N-1$ margins and 1 corner margin

3. Update the annealing weights

4. Find the greatest simplex based on the annealing weights

5. Bisect it through the longest segment by finding a new point on the operating boundary

6. Bisect all other simplexes for which this is the longest line segment

7. Calculate the solid angle subtended by each new simplex and update the Riemann sum

8. Until desired accuracy is achieved, goto (3)

Three points require further explanation: A) simplex formation and bisection, B) the algorithm is adaptive based on an annealing schedule, and C) the solid angle subtended by each simplex must be calculated.

\section{A. Bisection}

The first simplexes are formed in steps (1) and (2). The "corner margins" consist of all search vectors for which each dimension has a value of \pm 1 . There is one corner vector centered in each orthant. Many possibilities exist for subsequent iterations. Experimentation indicates that keeping the aspect ratio of each simplex small is important. This explains the conditional in step (6) of the algorithm.

The number of simplexes grows exponentially with $N$ and with iteration number, and becomes a limiting factor above 8-D. Integration in 7-D can create upwards of 500,000 simplexes; in 8-D, 5,000,000 simplexes. The problem is not storage per se, but the time spent doing unordered searches in steps (4) and (5). Incremental sorting is needed in step (4). Step (5) would benefit from an indexing system whereby each point had a field indicating its simplexes. Alternately, the simplexes could be organized by orthant. in this case, the signs of the coordinates for each point could be used as the index. The unordered searches in the current implementation limit us to about $8 \mathrm{D}$.

\section{B. Anneal}

Each term $\Omega_{s}\left\langle\mathrm{Q}_{s}\right\rangle$ in the Riemann sum is the product of the solid angle subtended by the simplex and the average value of $\mathrm{Q}$ for the points defining the simplex, The schedule computes the annealing weight of each simplex as $\Omega_{s}\left\langle\mathrm{Q}_{s}\right\rangle^{w}$. The schedule moves the value of $w$ from 0 to 1 incrementally. When $w=0$, the algorithm bisects the simplex with greatest solid angle; when $w=1$, the simplex that is most significant. In this way the algorithm starts by covering the space uniformly, and gradually becomes more adaptive. The anneal could be taken yet further by computing the variance among the values of the points in each simplex, and weight the anneal accordingly. This produced unstable results in our experimentation. However, we do compute and sum these variances in order to estimate the error, as for error estimates in Monte Carlo integration 32].

If the anneal proceeds too quickly, the danger is that it will find some local minima, while missing others of more significance. As stated previously, $3^{N}$ points may be needed to cover the space. However, we have found via a user-defined annealing parameter that completing the anneal with $2.5^{N}$ points can achieve reliable results.

\section{Solid Angles}

We need the solid angle subtended by each simplex, $\Omega_{s}$. (This is the language of three dimensions. For higher dimensions, this generalizes to the volume subtended on the unit hypersphere.) The solid angle is normalized by the whole, $\Omega_{N}=\pi^{N / 2} / \Gamma(N / 2+1)$, where $\Gamma$ is the gamma function. The procedure for calculating the solid angle of a simplex required some effort, as we invented our own.

First we calculate the volume, $V_{s}$, subtended by simplex $s$ on the operating region boundary surface, by also including the point at the origin. This volume derives from the matrix determinant. In $N$ dimensions

$$
V_{s}=\left|\frac{1}{N !} \operatorname{det}\left(v_{1}, v_{2}, \ldots, v_{N}\right)\right|
$$

where $\left(v_{1}, v_{2}, \ldots, v_{N}\right)$ are the unit vectors associated with the vertices of simplex $s$. The points at the center of each facet of simplex $s$ form a similar simplex. These points $\left(v_{1}^{\prime}, v_{2}^{\prime}, \ldots, v_{N}^{\prime}\right)$ are calculated by excluding the points of the simplex one-by-one and finding the center among the remaining points:

$$
\begin{aligned}
v_{1}^{\prime} & =\left\langle v_{2}, v_{3}, \ldots, v_{N}\right\rangle \\
v_{2}^{\prime} & =\left\langle v_{1}, v_{3}, \ldots, v_{N}\right\rangle \\
v_{N}^{\prime} & =\left\langle v_{1}, v_{2}, \ldots, v_{N-1}\right\rangle
\end{aligned}
$$

Here the angle brackets denote the mean value of the vector coordinates. The vertices of the new simplex lie inside the unit sphere. Renormalize them to unit vectors, and calculate the volume of this new simplex, $V_{s}^{\prime}$, again 
by including the point at the origin. The method centers on the observation that the volume of the new simplex is smaller by a factor of $V_{f}=1 /(N-1)^{(N-1)}$, in the limit of small angles. Scale inversely with $V_{f}$ to produce a new solid angle estimate

$$
V_{s}^{\prime}=(N-1)^{(N-1)}\left|\frac{1}{N !} \operatorname{det}\left(v_{1}^{\prime}, v_{2}^{\prime}, \ldots, v_{N}^{\prime}\right)\right|
$$

This produces a much better estimate for the original solid angle. Finally, we use both estimates to extrapolate to the best estimate,

$$
\Omega_{s}=\left(V_{s}^{\prime} / V_{s}\right)^{F_{N}} V_{s}^{\prime}
$$

where the first term produces a correction to $V_{s}^{\prime}$. The fitting parameter $F_{N}$ was found through experimentation.

$$
\begin{array}{r}
\left(F_{3}, F_{4}, \ldots, F_{10}\right)=(0.0000,-0.0997,-0.1106,-0.1057 \\
-0.0978,-0.0888,-0.0808,-0.0711)
\end{array}
$$

This method is effective because the correction to $V_{s}^{\prime}$ is small. Typical accuracy was better than $1 \%$, judged by summing the solid angles $\Omega_{s}$ and comparing to the known value of $\Omega_{N}$.

\section{ACKNOWLEDGMENTS}

The authors acknowledge valuable discussions with Alexander Sirota, Charles Wallace, and Henry Luo.
[1] Q. P. Herr, A. Y. Herr, O. T. Oberg, and A. G. Ioannidis, Journal of applied physics 109, 103903 (2011).

[2] D. S. Holmes, A. L. Ripple, and M. A. Manheimer, IEEE Transactions on Applied Superconductivity 23, 1701610 (2013).

[3] I. Vernik, A. Kirichenko, O. Mukhanov, and T. Ohki, IEEE Transactions on Applied Superconductivity 27, 1 (2016).

[4] A. Y. Herr, Q. P. Herr, O. T. Oberg, O. Naaman, J. X. Przybysz, P. Borodulin, and S. B. Shauck, Journal of Applied Physics 113, 033911 (2013).

[5] C. L. Ayala, T. Tanaka, R. Saito, M. N. Nozoe, N. Takeuchi, and N. Yoshikawa, IEEE journal of solidstate circuits in press (2021).

[6] Q. P. Herr and M. J. Feldman, Applied physics letters 69, 694 (1996).

[7] A. V. Rylyakov and K. K. Likharev, IEEE transactions on applied superconductivity 9, 3539 (1999).

[8] P. Bunyk and D. Zinoviev, IEEE transactions on applied superconductivity 11, 529 (2001).

[9] K. Fujiwara, N. Nakajima, T. Nishigai, M. Ito, N. Yoshikawa, A. Fujimaki, H. Terai, and S. Yorozu, IEEE transactions on applied superconductivity 15, 427 (2005).

[10] O. Wetzstein, T. Ortlepp, R. Stolz, J. Kunert, H.-G. Meyer, and H. Toepfer, IEEE transactions on applied superconductivity 21, 814 (2011).

[11] M. Tanaka, A. Kitayama, T. Takinami, Y. Komura, and A. Fujimaki, in 2013 IEEE 14th International Superconductive Electronics Conference (ISEC) (IEEE, 2013), pp. $1-3$.

[12] N. Takeuchi, H. Suzuki, and N. Yoshikawa, Applied Physics Letters 110, 202601 (2017).

[13] T. Filippov, Y. A. Polyakov, V. Semenov, and K. Likharev, IEEE Transactions on Applied Superconductivity 5, 2240 (1995).

[14] M. Klein and A. Mukherjee, Applied Physics Letters 40, 744 (1982).

[15] Q. P. Herr and M. J. Feldman, IEEE transactions on applied superconductivity 7, 2661 (1997).

[16] J. Satchell, IEEE transactions on applied superconductivity 9, 3841 (1999).
[17] A. B. Kahng, in 2015 52nd ACM/EDAC/IEEE Design Automation Conference (DAC) (IEEE, 2015), pp. 1-6.

[18] W. Press, S. Teukolsky, W. Vetterling, and B. Flannery, Numerical Recipes in $C$ 2nd edition (Cambridge University Press, 1992), chap. Incomplete Gamma Function.

[19] S. Director and G. Hachtel, IEEE Transactions on Circuits and Systems 24, 363 (1977).

[20] Q. P. Herr and M. W. Johnson, IEEE transactions on applied superconductivity 11, 1078 (2001).

[21] A. L. Braun and D. C. Harms, RQL Majority gates, And gates, and Or gates (2018), US Patent 10,084,454.

[22] A. L. Braun, Superconducting devices with enforced directionality (2018), US Patent 10,153,772.

[23] S. Whiteley, XicTools: Xic graphical editor, WRspice circuit simulator, and accessories for electronic design (https://github.com/wrcad/xictools, 2020).

[24] A. Berkley, M. Johnson, P. Bunyk, R. Harris, J. Johansson, T. Lanting, E. Ladizinsky, E. Tolkacheva, M. Amin, and G. Rose, Superconductor Science and Technology 23, 105014 (2010).

[25] D. C. Harms, Q. P. Herr, and A. Y. Herr, Superconducting circuits based devices and methods (2019), US Patent $10,411,713$.

[26] K. K. Likharev, Dynamics of Josephson junctions and circuits (Gordon and Breach science publishers, 1986), chap. The DC Josephson Effect.

[27] E. Harris, IEEE Transactions on Magnetics 15, 562 (1979).

[28] D. V. Averin, K. Rabenstein, and V. K. Semenov, Physical Review B 73, 094504 (2006).

[29] E. Fredkin and T. Toffoli, International Journal of theoretical physics 21, 219 (1982).

[30] Q. P. Herr, J. E. Baumgardner, and A. Y. Herr, Method and apparatus for ballistic single flux quantum logic (2010), US Patent 7,782,077.

[31] K. D. Osborn and W. Wustmann, IEEE Transactions on Applied Superconductivity 31, 1 (2020).

[32] W. Press, S. Teukolsky, W. Vetterling, and B. Flannery, Numerical Recipes in $C$ 2nd edition (Cambridge University Press, 1992), chap. Simple Monte Carlo Integration. 